\title{
miR-338-5p Regulates the Viability, Proliferation, Apoptosis and Migration of Rheumatoid Arthritis Fibroblast-Like Synoviocytes by Targeting NFAT5
}

Ting Guo Hao Ding Hui Jiang Nirong Bao Liwu Zhou Jianning Zhao

Department of Orthopaedics, Jinling Hospital, Nanjing, China

\section{Key Words}

Rheumatoid arthritis $・$ RAFLSs $・$ MiR-338-5p • NFAT5

\begin{abstract}
Background/Aims: MicroRNAs (miRNAs) have been reported to be involved in Rheumatoid arthritis (RA) pathogenesis and prognosis. However, little is known about the disease mechanism in RA. Here, we aim to investigate the potential association between miR-338-5p and NFAT5 in RA. Methods: Aberrant expression of miR-338-5p in RA tissues and rheumatoid arthritis fibroblast-like synoviocytes (RAFLSs) compared to the normal were determined by RT-qPCR. Cell viability was determined using the CCK- 8 assay, and cell apoptosis was analyzed via Annexin V-FITC/PI double staining and was detected using flow cytometry. The targeted relationship was determined by TargetScan database and dual luciferase reporter gene assay. Results: Upregulation of miR-338-5p facilitated the proliferation, migration, invasion and induced G0/G1 arrest of RAFLSs while miR-338-5p inhibitor functioned oppositely. Nuclear factor of activated T-cells 5 (NFAT5) was confirmed as a downstream target of miR338-5p which expression was directly suppressed by miR-338-5p. Overexpression of NFAT5 attenuated the proliferation and metastasis of RAFLSs and those changes could be rescued by co-transfection of miR-338-5p. Conclusion: miR-338-5p promotes RAFLS's viability and proliferation, migration by targeting NFAT5, suggesting a novel therapeutic strategy for RA.
\end{abstract}

\section{Introduction}

Rheumatoid arthritis (RA) is a chronic autoimmune disorder that is characterized by propagation of synovial tissue and mainly attacks the joints [1, 2]. RA has complex pathogenesis and unpredictable prognosis [3]. It is widely believed that rheumatoid arthritis fibroblast-like synoviocytes (RAFLSs) play key roles in RA progression [4-6]. RAFLSs not only passively respond to the regulations from T-cells, B-cells and macrophages, but also 


\section{Cellular Physiology Cell Physiol Biochem 2018:49:899-910

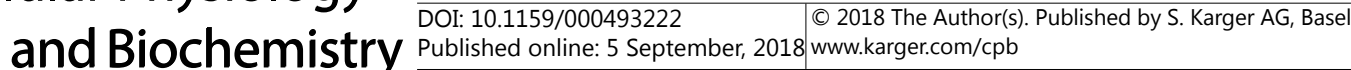 \\ Guo et al.: miR-338-5p Targets NFAT5 and Influences Raflss}

actively participate in chronic inflammation of synovial joints which lead to cartilage and bone destruction [7]. An important feature of synovial fibroblasts in RA is the oversecretion of matrix-degrading enzymes and cytokines/chemokines, which then attracts and activated immune cells that stimulate synovial fibroblasts that in turn, resulting in chronic joint inflammation [4, 7]. The inhibition of cytokines/chemokines such as IL-6, IL-29 and TNF- $\alpha$ etc. could reduce RA clinical symptoms [8], IL-35 might delay RA progression through regulation of the VEGF/Ang2 crosstalk [9], which explained the critical role of cytokines in RA $[10,11]$.

To clarify the pathogenesis mechanism of RA, a recently discovered class of genes transcribing small non-coding RNAs, namely microRNAs, comes into focus, which could be potential serving as new therapeutic strategies for cancers. MicroRNAs are small noncoding RNAs that target specific 3' UTR of mRNAs and thereby degrade targeted mRNAs or hinder the mRNA translation. It was stated that miRNAs have been potential regulators in various diseases $[12,13]$. Altered miRNA expression has been reported to be involved in the pathogenesis of RA $[14,15]$. For instance, the roles of miR-155 and miR-146a have been thoroughly studied in RA [16], and the elevated expression of the two miRNAs has been reported by Filkova et al. and Ceribelli et al. [14, 17]. Here, we found miR-338-5p was significantly enriched in RA tissues by microarray analysis. A few studies have discovered the role of miR-338-5p in various disease progressions, such as neural degeneration, an early biomarker of cancers and mitochondrial toxicity [18-22]. MiR-338-5p was also reported as an immunity-related molecule and RA development was partly attributed to immunity disorder [23-25]. Hence, it was hypothesized that miR-338-5p might exert a strong influence on aggravation of RA.

By coupling gene expression profiling of synovial tissues and the online database TargetScan Human 7.0, we predicted nuclear factor of activated T-cells 5 (NFAT5) as a potential target gene of miR-338-5p with the binding sequence ATATTGT in its 3'UTR. NFAT5 plays a central role in inducible gene transcription during the immune response and regulates gene expression induced by osmotic stress in mammalian cells [26]. Masuda K et al. revealed that NFAT5 was primarily expressed in synovial tissues in RA rather than those in normal individuals [27]. Kim NH et al. discovered that the xanthine oxidase-NFAT5 pathway regulated macrophage activation and TLR-induced arthritis and the down-regulation of xanthine oxidase-NFAT5 was associated with the less severe arthritis [26]. NFAT5 could indirectly down-regulate miR-140 by up-regulating TGF- $\beta$ and subsequently activating SMAD3 in osteoarthritis [28]. NFAT5 has been found to be active in carcinoma invasion and RA $[29,30]$. In addition, Yoon HJ et al. found that NFAT5 was overexpressed in rheumatoid synovium and that NFAT5 could regulate the synovial propagation and angiogenesis in RA [30].

In the present study, we found an extremely high expression of miR-338-5p and low expression of NFAT5 in RA tissues and RAFLSs compared to the normal. We also unraveled that miR-338-5p/NFAT5 axis could regulates the biological functions of RAFLSs including cell viability, migration, invasion, cell cycle and apoptosis in vitro, which is helpful to develop meaningful strategies for RA treatment.

\section{Materials and Methods}

Tissue samples

The synovial tissues from 60 RA patients were recruited during joint surgery in Jinling Hospital from 2014 to 2016.17 males and 43 females were included and the mean age was 49 years old. Healthy control synovial tissue samples were obtained from 30 emergent trauma amputation patients consists of 11 males and 19 females; the mean age is 44 years old (Table 1). All healthy control specimens were free from RA or osteoarthritis. Written permission was obtained from all participants in the study. The ethics committee's approval was granted by Jinling Hospital. 


\section{Cellular Physiology Cell Physiol Biochem 2018;49:899-910

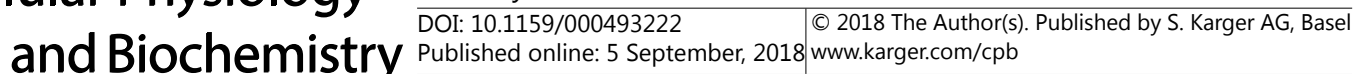

RAFLSs and healthy control cells culture

RA samples and healthy control samples were cut into small pieces, washed with Hank's solution and then digested with trypsin solution. Collected cells were cultured in the inverted Dulbecco's Modified Eagle Medium (DMEM, Invitrogen, Carlsbad, USA) culture flasks until cell reached $70-80 \%$ confluences and then RAFLSs and healthy control cells were separated using fluorescence activated cell sorting technique. Isolated RAFLSs and healthy control cells were maintained in DMEM with $10 \%$ fetal bovine serum (FBS) at $37{ }^{\circ} \mathrm{C}$ within $5 \% \mathrm{CO}_{2}$.

\section{Microarray assay}

Total RNA from 4 RA tissues and 4 healthy control synovial tissues were prepared using Trizol reagent ( Thermo Fisher Scientific, Waltham, USA). After purified by RNase free DNase, total RNA was quantified by NanoDrop 2000 spectrophotometer. $100 \mathrm{ng}$ of total RNA was treated with Low Input Quick Amp Labeling kit (Agilent 5190-2305, Agilent Technologies, Santa Clara, USA) or miRNA Complete Labeling and Hyb kit (Agilent Technologies) according to the manufacturer's instruments. SurePrint G3 Human V21.0 Gene (Agilent Technologies) and MiRNA Microarray (Agilent Technologies) were utilized by Biotechnology Co. Shanghai to assess the gene and miRNA expression patterns respectively. The system contains a large number of probes for 2549 miRNAs and 47000 mRNAs. The intensity data were obtained with the Feature Extraction 10.7 software (Agilent Technologies). All digitized data were analyzed using R language.

\section{$R T-q P C R$}

Tissue samples were torn into pieces, and then total RNA was extracted using $1 \mathrm{ml}$ TRIzol reagent (Thermo Fisher Scientific) for every 30-50mg tissue samples. Separated RNA was then purified and quantified as previously described. For miRNA measurement, reverse transcription was performed using the Taqman MicroRNA Reverse Transcription Kit (Life Technologies, Gaithersburg, USA). QuantiFast SYBR Green PCR kit (Qiagen, Duesseldorf, Germany) was applied to acquire corresponding cDNA for mRNA analysis. Afterwards, real-time PCR was performed using an 7900HT Sequence Detection System (Applied Biosystems, USA). Primer sequences were shown in Table 2. The fold changes of miRNA and mRNA were calculated using $2^{-\triangle \Delta C T}$ method using the level of U6 and GAPDH as the internal control, respectively.

\section{Cell transfection}

MiR-338-5p mimic, inhibitor and corresponding control sequences (Negative control 11 means miR338-5p mimic control, and negative control 12 means miR-338-5p inhibitor control) were purchased from Sigma-Aldrich, Shanghai. NFAT5 cDNA was synthesized by Genepharma Co., Shanghai, and was inserted into pcDNA3.1 plasmid. RALFs were first plated in a medium without antibiotics. After cells reached $60 \%$ confluency, transfections were performed with Lipofectamine 2000 in accordance with manufacturer's protocol.
Table 1. The pathological characteristics of patients in RA group and normal group. RA: Rheumatoid arthritis; CRP: C-reactive protein; ESR: erythrocyte 28; RF: rheumatoid factor; anti-CCP: anti-cyclic citrullinated proteins antibodies; na: not available. * represents that $P$ value was analyzed by Mann-Whitney

Table 2. Primer sequences of Real-time PCR

\begin{tabular}{lll}
\hline cDNA & Forward & Reverse \\
\hline Mir-338-5p & 5'-GAGCTCCGAGCAATTCTCCTGTGTC-3' & 5'-CTCGAGTACAGCATCCCTCCCAAC-3' \\
NFAT5 & 5'-ATCATCAGCAACAGGTCAG-3' & 5'-GGAAGGAATCATCACTATACAG-3' \\
U6 & 5'-CTCGCTTCGGCAGCACA-3' & 5'-AACGCTTCACGAATTTGCGT-3' \\
GAPDH & 5'-TGACTTCAACAGCGACACCCA-3' & 5'-CACCCTGTTGCTGTAGCCAAA-3' \\
\hline
\end{tabular}

\begin{tabular}{llll}
\hline Index & Normal & RA & P value \\
\hline Age & $44.4 \pm 16.4$ & $48.8 \pm 11.0$ & $/$ \\
Gender & & & $/$ \\
$\quad 11$ & 17 & \\
$\quad$ Male $\quad$ Female & 19 & 43 & \\
CRP (mg/l) & na & $21.5 \pm 3.5$ & $/$ \\
ESR (mm/h) & $5.7 \pm 1.1$ & $30.0 \pm 5.0$ & $P<0.001^{*}$ \\
DAS28 & na & $4.9 \pm 1.0$ & $/$ \\
RF positive (\%) & na & $75.4 \pm 13.7$ & $/$ \\
Anti-CCP positive $(\%)$ & $6.4 \pm 1.3$ & $77.8 \pm 11.2$ & $P<0.001^{*}$ \\
\hline
\end{tabular}




\section{Cellular Physiology Cell Physiol Biochem 2018;49:899-910

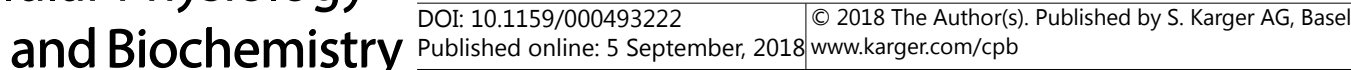 \\ Guo et al.: miR-338-5p Targets NFAT5 and Influences Raflss}

\section{Dual luciferase reporter gene assay}

The wild type and a mutant 3'UTR sequence of NFAT5 which contained the putative miR-338-5p binding sites were inserted into the pMIR-REPORT Luciferase vectors (Ambion, Austin, USA) to construct pMIR/ NFAT5-wt and pMIR/NFAT5-mut vectors. RAFLSs were collected after co-transfection of pMIR/NFAT5-wt and pMIR/NFAT5-mut with miR-338-5p mimic or miR-338-5p mimic control using Lipofectamine 2000, respectively. Subsequently PLB (Passive Lysis Buffer) was added as well as the plate was gently shaken for 15 minutes at room temperature, and then cell lysates were collected. $50 \mu \mathrm{L}$ LAR II and $10 \mu \mathrm{L}$ cell lysate were added to an EP tube (Eppendorf tube, $0.5 \mathrm{~mL}$ ), the luciferase intensity RLU1 was obtained. Thereafter $50 \mu \mathrm{L}$ Stop \& Glo ${ }^{\circledR}$ Reagent will be added to the EP tube (Eppendorf tube, $0.5 \mathrm{~mL}$ ) and the luciferase intensity RLU2 will be obtained. The relative fluorescence intensity ratio will then be presented as the ratio of RLU1/RLU2.

\section{Western Blot}

Total proteins were obtained after lysing on ice-cold RIPA buffer (Solaibo, Beijing, China) and then quantified by bicinchoninic acid. Thereafter, electrophoresis on sodium dodecyl sulfate, sodium salt polyacrylamide gel electrophoresis (SDS-PAGE) gel was performed and then proteins were transferred to the polyvinylidene fluoride (PVDF) membrane and incubated at room temperature for 30 60 minutes. After the 5\% low-fat milk solution blocking (1h), primary antibodies were added and incubated overnight at $4{ }^{\circ} \mathrm{C}$. Then the membranes were washed with Tris Buffer Solution Tween (TBST) and secondary antibodies were then added and incubated at room temperature for $1 \mathrm{~h}$. All antibodies were purchased from Abcam (Cambridge, USA) including ab105886, ab 8227 and ab 97080. Lastly, the Electro-Chemi-Luminescence (ECL) solution was applied and the images were photographed using Bio Doc It (UVP Co, USA) to be analyzed for densitometry by Lab Works 4.5 software.

\section{CCK-8 assay}

The Cell Counting Kit-8 assay was performed strictly following corresponding instructions. Briefly, transfected RAFLSs were seeded at $1 \times 10^{4} /$ well and cultured for 24,48 and 72 hours. CCK-8 kit $(10 \mu \mathrm{L}$, Beyotime, Shanghai, China) was added at each time point and the incubation was sustained for 4 hours before the OD values at $450 \mathrm{~nm}$ was determined by a microplate reader (Thermo Fisher Scientific, Waltham, USA). All experiments were done in triplicate and the averages were used for statistical analysis.

\section{Flow cytometry analysis}

RAFLSs were collected at 48 hours after transfection and then digested with trypsin. Flow cytometry analysis was carried through Cell Cycle and Apoptosis Analysis Kit (Beyotime, Shanghai, China). Cells were added to $1 \mathrm{~mL}$ pre-cooling $70 \%$ ethanol, gently blowing mix, $4{ }^{\circ} \mathrm{C}$ fixed 2 hours. For cell cycle analysis, cells were re-suspended in prepared sample buffer containing $20 \mu \mathrm{L} 5 \mu \mathrm{g} / \mathrm{mL}$ propidium iodide and $50 \mu \mathrm{L} 10$ $\mathrm{mg} / \mathrm{mL}$ RNase A and then incubated in the dark for $10 \mathrm{~min}$ at $37^{\circ} \mathrm{C}$. In cell apoptosis analysis, the cells were re-suspended in binding buffer ( $\mathrm{pH}$ 7.4) including $100 \mathrm{mM}$ 4-(2-hydroxyethyl)-1-piperazineethanesulfonic acid (HEPES), $100 \mathrm{mM} \mathrm{NaCl}$, and $25 \mathrm{mM} \mathrm{CaCl2}$. Lastly, 0.3ml Annexin V-FITC/PI were added to the EP tubes, the cells were incubated for $15 \mathrm{~min}$ at $37^{\circ} \mathrm{C}$. After adding $400 \mu \mathrm{L}$ binding buffer into each tube, cells in different phases and apoptosis rate were detected using flow cytometry (BD Biosciences, USA).

\section{Transwell assay}

Transwell chambers were prepared with or without Matrigel for evaluate the invasive and migratory abilities of transfected RAFLSs respectively. Cells $\left(2.5 \times 10^{4}\right)$ were seeded in upper chambers and cultured at $5 \% \mathrm{CO}_{2}, 37^{\circ} \mathrm{C}$ for $48 \mathrm{~h}$. The upper chambers were cleaned with sterilized cotton swab to remove the cells attached to the Matrigel on the chambers side. Then the lower membrane was fixed in $4 \%$ paraformaldehyde and stained with $0.1 \%$ crystal violet. The numbers of stained cells in five random fields were counted lastly.

\section{Statistical analysis}

The measurement data were presented as mean \pm standard deviation $(\bar{X} \pm s)$. Students' t-test was used to compare normally distributed measurement data between any two groups. And the differences among multiple groups were obtained via one-way ANOVA. All data analysis was performed by GraphPad Prism 6.0, and $P<0.05$ was considered statistically significant. 


\section{Results}

Aberrant expression of miR-338-5p in $R A$ synovial tissues and RAFLSS

MicroRNA microchip assay was first performed to identify significant differences in microRNA expression patterns between RA synovial tissues and healthy control. Six miRNAs were found differently expressed in RA tissues compared with the control $(P<0.01$, fold change $>2$, Fig. $1 \mathrm{~A}$ and 1B, Table 3). Among the six miRNAs, miR-338-5p showed smallest $P$ value and was selected for further research. Then we detected the expression level of miR-338-5p on 60 RA synovial tissues and 30 healthy control samples using RT-qPCR. As shown in Fig. 1C, miR-338-5p was remarkably enriched in RA tissues $(P<0.05)$. Further, we separated RAFLSs from RA synovial tissues and normal cells from healthy control tissues. RTqPCR further confirmed the high level of miR-338-5p in RAFLSs $(P<0.05$, Fig. 1D). Hence, we predicted that the aberrant expression of miR-338-5p was correlated to the progression of RA.

\section{Regulation of miR-338-5p on biological behaviors in RAFLSS}

To investigate the potential
B
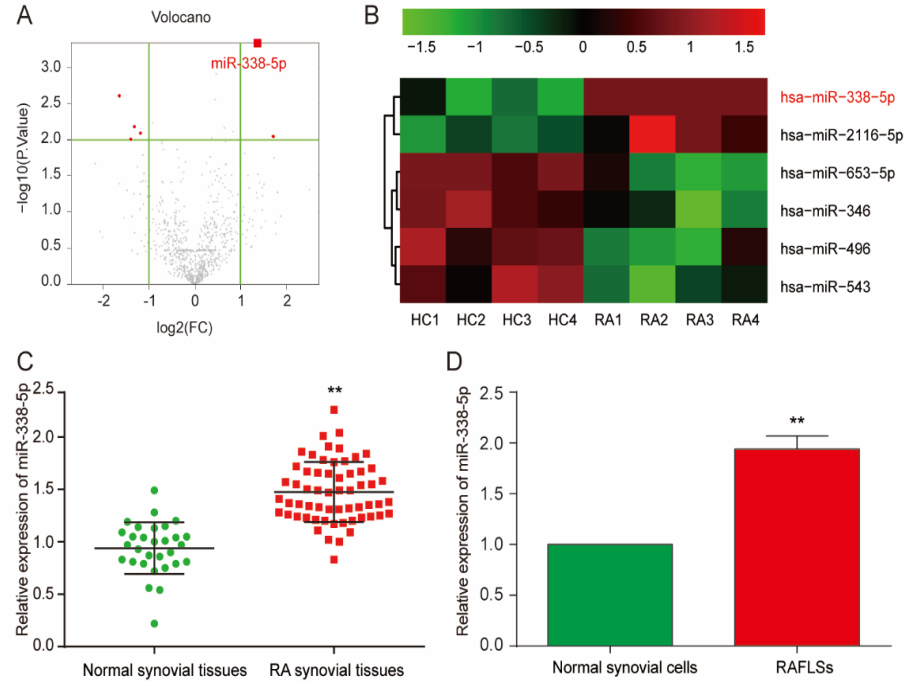

$\mathrm{D}$

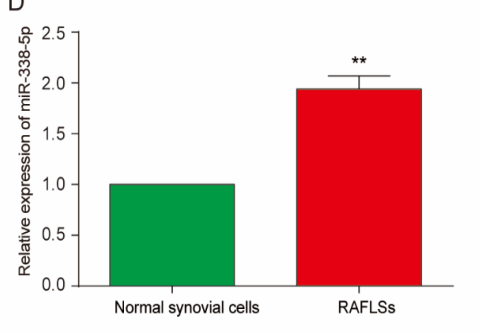

Fig. 1. Aberrant expression of miR-338-5p in RA synovial tissues and RAFLSs. (A) MiRNA microarray analysis was performed using 4 RA synovial tissues and 4 healthy control. Volcano plots of differentially expressed miRNAs were shown. Six miRNAs with a fold change more than 2 and $P$ value less than 0.01 were selected. (B) Heat map of miRNA expression profiles was shown. Red color represents upregulation and green color indicates downregulation. (C) Expression of miR-338-5p in 60 RA synovial tissues and 30 healthy control tissues was detected by RT-qPCR. (D) RAFLSs and healthy control cells were separated. RTqPCR was utilized to value the expression of miR-338-5p. Error bar indicates mean values \pm SD. HC means healthy control, the scale bar for Fig. 1B means Z-scores. ${ }^{* *} \mathrm{P}<0.01$.

Table 3. Differentially expressed microRNAs in RA synovial tissues compared with the healthy control were selected via miRNA microarray analysis

\begin{tabular}{lllllllll}
\hline microRNA & RA1 & RA2 & RA3 & RA4 & OA1 & OA2 & OA3 & OA4 \\
\hline hsa-miR-338-5p & 35.0000 & 35.0000 & 35.0000 & 35.0000 & 34.2250 & 33.3093 & 33.7062 & 33.3187 \\
hsa-miR-653-5p & 34.30188 & 33.1550 & 32.7562 & 32.9375 & 35.0000 & 35.0000 & 34.6900 & 35.0000 \\
hsa-miR-496 & 30.83188 & 30.6325 & 30.4750 & 31.8287 & 32.7250 & 31.8850 & 32.2056 & 32.2887 \\
hsa-miR-543 & 26.9612 & 26.3087 & 27.1812 & 27.4487 & 28.0425 & 27.6162 & 28.5950 & 28.3462 \\
hsa-miR-2116-5p & 32.91188 & 35.0000 & 33.9200 & 33.4637 & 31.7312 & 32.4700 & 32.0187 & 32.3356 \\
hsa-miR-346 & 33.3037 & 33.1037 & 31.6925 & 32.46813 & 34.1337 & 34.4650 & 33.8762 & 33.6775 \\
microRNA & \multicolumn{2}{c}{ logFC } & AveExpr & t & & P.Value \\
hsa-miR-338-5p & 1.36015625 & 34.31993188 & -6.52973376 & 0.000457944 \\
hsa-miR-653-5p & -1.63484375 & 34.10508813 & 4.756621589 & 0.00258202 \\
hsa-miR-496 & -1.3340625 & 31.6090725 & 3.94863199 & 0.006529291 \\
hsa-miR-543 & -1.175 & 27.56251 & 3.782836437 & 0.007994723 \\
hsa-miR-2116-5p & 1.685 & 32.98141625 & -3.694491347 & 0.008920825 \\
hsa-miR-346 & -1.39609375 & 33.34008813 & 3.60838144 & 0.009937997 \\
\hline
\end{tabular}

role of miR-338-5p on the growth of RAFLSs, we first manipulated the expression of miR-338$5 p$ in RAFLSs by transient transfection. RAFLSs were randomly divided into five groups and transfection was performed in each group using Lipofectamine 2000. Cells were collected at 48 hours after transfection. Accessed by RT-qPCR (Fig. 2A), transfection of negative control 1 and negative control 2 had little effect on the expression of miR-338-5p compared to the blank control in which group RAFLSs were untreated. And miR-338-5p mimic promoted the expression of miR-338-5p while miR-338-5p inhibitor effectively downregulated its 
Fig. 2. Regulation of miR-338-5p on proliferation, migration and invasion of RAFLSs. (A) At 48 hours after transfection, RAFLSs were collected and expression of miR-338-5p was measured by RTqPCR. (B) Effects of miR-338-5p mimic and miR338-5p inhibitor on proliferation of RAFLSs were evaluated using CCK8 assay. (C) Transwell assay was performed to investigate effects of miR-338$5 \mathrm{p}$ expression on cell migratory and invasive abilities. (D-

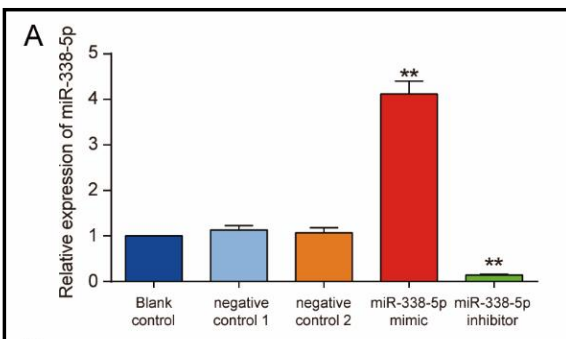

C
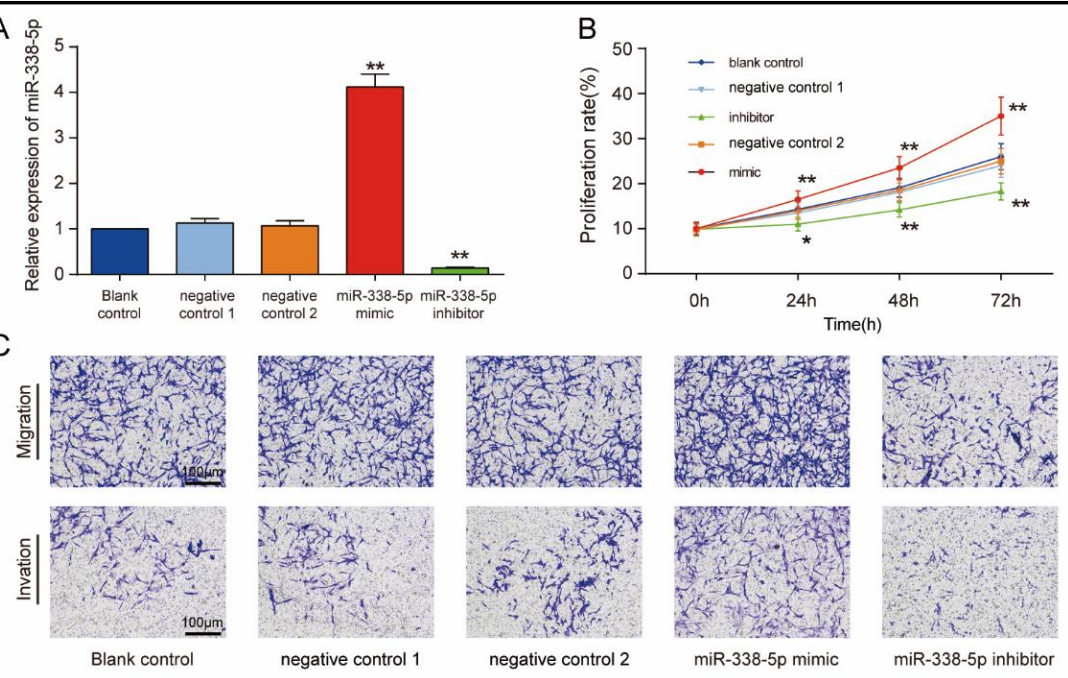

$D$

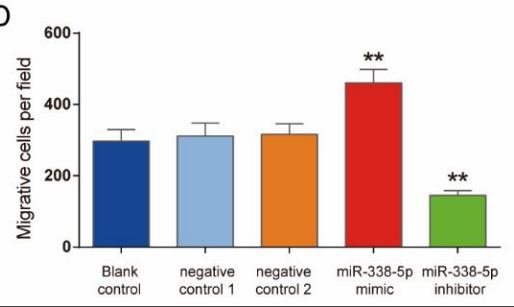

E

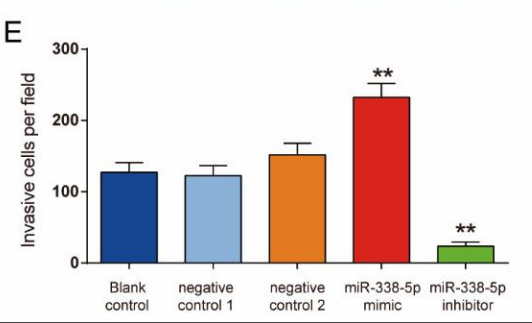

E) Cell number in five random fields was counted and quantitation of RAFLSs' migration and invasion was made. Error bar indicates mean values of triplicate experiments \pm SD. Negative control 1 means miR-338$5 p$ mimic control, Negative control 2 means miR-338-5p inhibitor control. ${ }^{*} \mathrm{P}<0.05,{ }^{* *} \mathrm{P}<0.01$ compared with relative control group.

Fig. 3. Regulation of miR-338-5p on cell cycle and apoptosis of RAFLSs. (A) After $P I$ and Annexin V-FITC staining, effects of miR-338$5 \mathrm{p}$ mimic and miR338-5p inhibitor on apoptosis of RAFLSs were detected by flow cytometry. (B) Transfected cells were stained with PI and cell cycle was measured using flow cytometry. (CD) Histogram was drawn to quantify cell cycle and cell
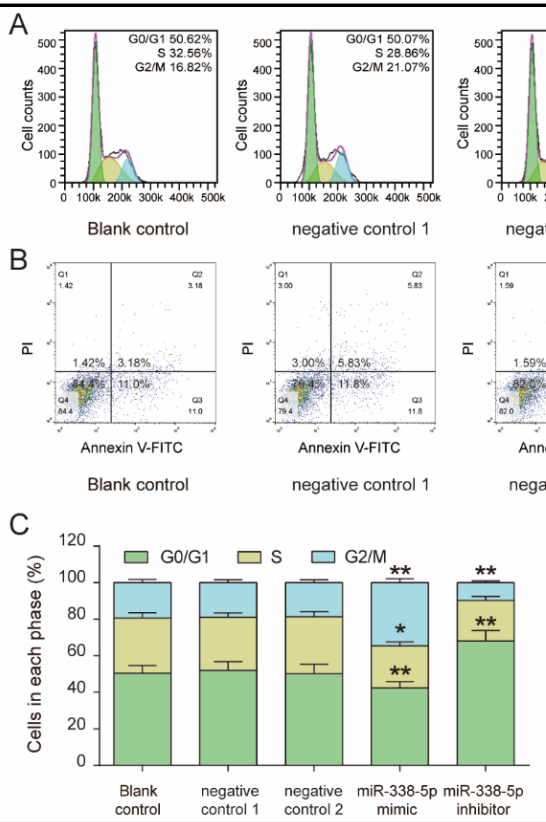
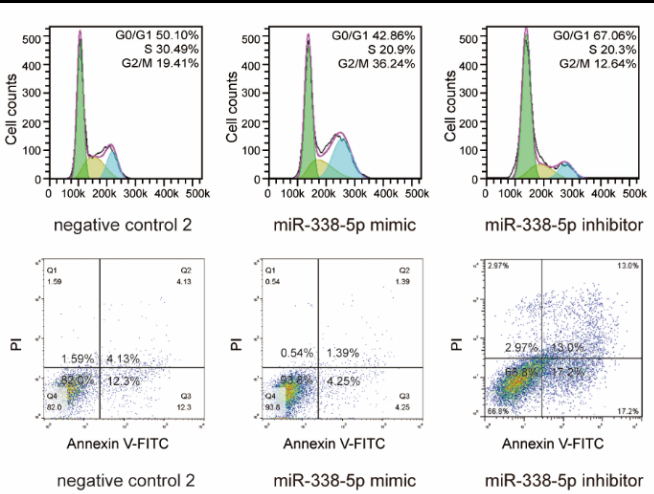

D

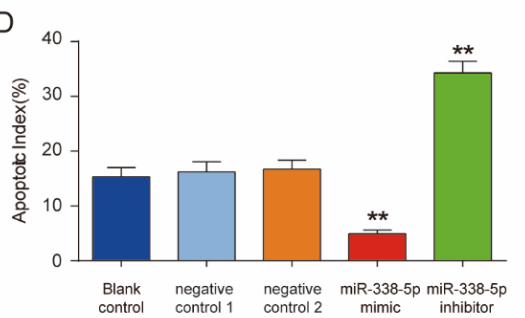
apoptosis rate in different groups. Error bar indicates mean values of triplicate experiments \pm SD. ${ }^{*} \mathrm{P}<0.05,{ }^{* *} \mathrm{P}<0.01$ compared with relative control group. 
Fig. 4. Identification of NFAT5 as a miR-338-5p target. (A) Gene microarray analysis was performed using previous eight tissues. Volcano plots of differentially expressed genes (fold change $>4$ and $P$ value $<0.0001$ ) were shown. (B) Heat map of 12 selected genes was shown. Red color represents upregulation and green color indicates downregulation. (C) Sequence alignment of miR-338$5 p$ and 3'-UTR of NFAT5 was illustrated. (D) Dual luciferase reporting assay was performed. RALFs were co-transfected with miR-338-5p mimic ornegative control 1 and a luciferase reporter containing either wild type or mutant type of NFAT5 3'UTR. Relative luciferase activities were detected. (E-F) MRNA expression and protein level of NFAT5 in
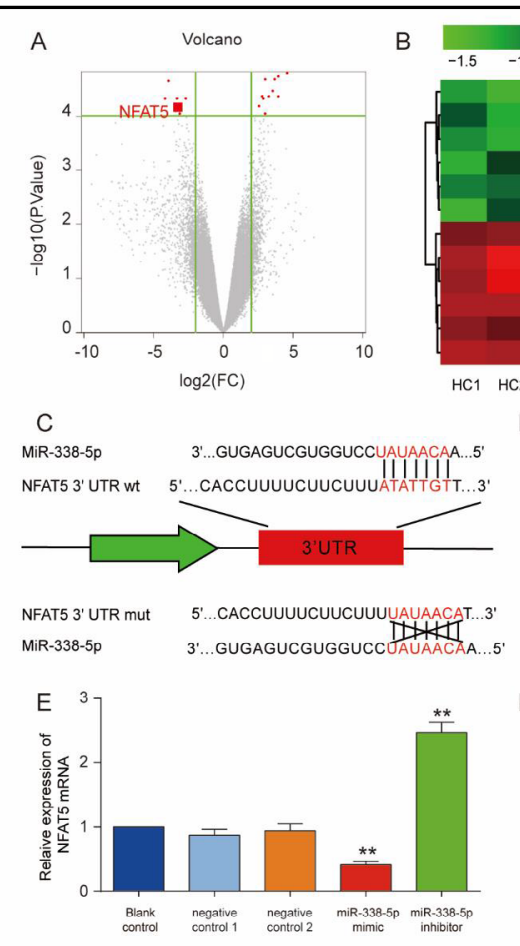

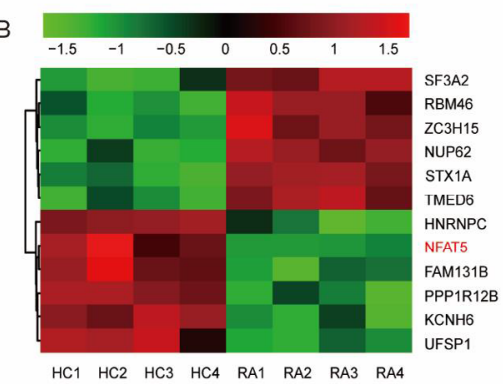

D

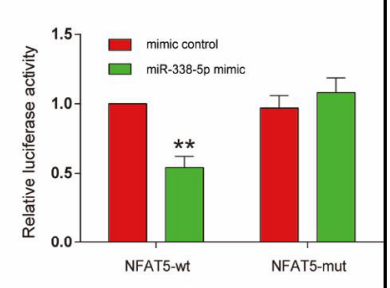

$\mathrm{F}$

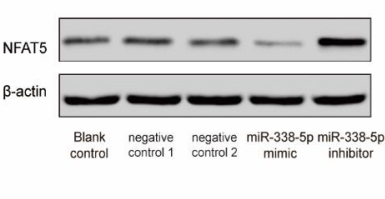
transfected cells were measured.

Error bar indicates mean values of triplicate experiments \pm SD. HC means healthy control, the scale bar for Fig. 1B means Z-scores. ${ }^{* *} \mathrm{P}<0.01$.

expression $(P<0.05)$. Cell proliferation, migration, invasion, cell cycle and apoptosis of transfected RAFLSs was subsequently examined.

Detected by CCK-8 assay, overexpressed miR-338-5p increased the cell proliferation which was attenuated in inhibitor group compared to corresponding control group $(P$ $<0.05$, Fig. 2B). However, RASFSs in negative control 1 and negative control 2groups showed similar growth curves with cells in blank group. Measured by Transwell assay, promoted migration and invasion of transfected RAFLSs was also found in mimic group compared with negative control 1 while migratory and invasive cells decreased in inhibitor group compared with negative control $2(P$ $<0.05$, Fig. 2C-2E). Downregulation of miR-338-5p also induced G0/G1 arrest and increased cell apoptosis rate while overexpression of miR-338$5 \mathrm{p}$ functions oppositely $(P<0.05$, Fig.
Table 4. Differentially expressed mRNAs in RA synovial tissues compared with the healthy control were presented via gene microarray analysis

\begin{tabular}{lllllllll}
\hline Genesymbol & HC1 & HC2 & HC3 & HC4 & RA1 & RA2 & RA3 & RA4 \\
\hline FAM131B & 4.45171 & 5.29933 & 3.95339 & 3.81138 & 1.04630 & 0.42598 & 1.73948 & 1.62008 \\
HNRNPC & 4.43499 & 4.65983 & 4.75163 & 4.94872 & 1.99686 & 0.97470 & -0.67554 & -0.15199 \\
KCNH6 & 3.75721 & 3.37004 & 4.34464 & 3.91689 & 0.66627 & 0.22061 & 1.51207 & -0.15199 \\
NFAT5 & 5.42495 & 6.25649 & 4.26304 & 4.66181 & 1.78387 & 1.78387 & 1.80915 & 2.01870 \\
NUP62 & 0.13466 & 1.77106 & 0.12607 & 0.20437 & 4.90658 & 4.52056 & 3.91565 & 4.45400 \\
PPP1R12B & 6.40444 & 6.34734 & 5.84178 & 5.50358 & 1.61599 & 3.13893 & 2.33939 & 1.13466 \\
RBM46 & -0.27562 & -1.73692 & -1.29856 & -2.03808 & 4.07598 & 3.28830 & 3.33691 & 2.11452 \\
SF3A2 & 1.17132 & 0.61804 & 0.71676 & 2.65535 & 4.81406 & 4.64285 & 5.68333 & 5.68979 \\
STX1A & 2.70622 & 2.95784 & 2.15451 & 1.90689 & 5.42991 & 5.59356 & 5.65535 & 5.16049 \\
TMED6 & 0.30934 & 1.61190 & 0.89935 & 0.30934 & 3.77839 & 4.32162 & 4.56350 & 3.49325 \\
UFSP1 & 4.56364 & 4.46668 & 4.83959 & 2.84057 & 0.42598 & 0.32945 & 1.33939 & 0.81985 \\
ZC3H15 & 1.24991 & 0.92601 & 1.31691 & 1.09703 & 4.29502 & 3.34557 & 3.72532 & 3.38873 \\
Genesymbol & logFC & & AveExpr & t & & P.Value \\
FAM131B & -3.170992382 & 2.793456734 & 6.574978193 & $9.23 \mathrm{E}-05$ \\
HNRNPC & -4.162785328 & 2.617400436 & 7.149505894 & $4.79 \mathrm{E}-05$ \\
KCNH6 & -3.285456997 & 2.204469289 & 7.171162433 & $4.68 \mathrm{E}-05$ \\
NFAT5 & -3.302672742 & 3.500234188 & 6.914392414 & $6.24 \mathrm{E}-05$ \\
NUP62 & 3.890156228 & 2.504118972 & -8.051358708 & $1.84 \mathrm{E}-05$ \\
PPP1R12B & -3.967043224 & 4.040763709 & 7.863534064 & $2.23 \mathrm{E}-05$ \\
RBM46 & 4.541216777 & 0.933315772 & -8.242390296 & $1.52 \mathrm{E}-05$ \\
SF3A2 & 3.917136454 & 3.248939136 & -7.214061907 & $4.46 \mathrm{E}-05$ \\
STX1A & 3.028460829 & 3.945596359 & -7.927080722 & $2.09 \mathrm{E}-05$ \\
TMED6 & 3.256708106 & 2.410835671 & -7.258258563 & $4.25 \mathrm{E}-05$ \\
UFSP1 & -3.448951838 & 2.453144884 & 6.666579754 & $8.29 \mathrm{E}-05$ \\
ZC3H15 & 2.541194676 & 2.418061918 & -6.891287167 & $6.40 \mathrm{E}-05$ \\
\hline
\end{tabular}
3A-3D). No significant differences were found among blank control, negative control 1 and negative control 2 groups in the four assays. Collective results suggested that miR-338-5p 


\section{Cellular Physiology Cell Physiol Biochem 2018;49:899-910 and Biochemistry Published 10,159/000493222 2018 ( 2018 The Author(s). Published by S. Karger AG, Basel \\ Guo et al.: miR-338-5p Targets NFAT5 and Influences Raflss}

promotes the proliferation, migration, invasion and blocked cellular progression from $\mathrm{G} 2 / \mathrm{M}$ into G0/G1 of RAFLSs.

\section{Identification of NFAT5 as a miR-338-5p target}

To find target genes of miR-338-5p, we first investigated gene expression profile in RA tissues and healthy control tissues. Twelve genes were selected and presented in Fig. 4A and 4B $(P<0.0001$, Fold change $>4$, Table 4). Then we utilized TargetScan and found 4810 transcripts which contains predicted target sites for miR-338-5p. Venn analysis was then carried out and only NFAT5 remained in the intersectional area of 12 genes and 4810 potential targets. The target relationship was then confirmed by dual luciferase reporting assay. The luciferase reporting constructs (pMIR-REPORT) was generated which contained either wild type or mutant 3'UTR of NFAT5. The mutant 3'UTR of NFAT5 differs from the wild type at 3400-3406 position of NFAT5 which was potential binding sites predicted by TargetScan (Fig. 4C). Co-transfection was performed in RAFLSs and luciferase activities were detected. As shown in Fig. 4D, the luciferase activity of cells transfected with both pMIRNFAT5 wt and miR-338-5p mimic significantly decreased compared with cells in pMIRNFAT5 wt + negative control 1 group $(P<0.05$, Fig. 4D). Meanwhile, cells transfected with pMIR-NFAT5 mut + miR-338-5p mimic and pMIR-NFAT5 mut + negative control 1 showed similar luciferase activity compared with pMIR-NFAT5 wt + negative control 1 group. Above data suggested that NFAT5 3'UTR region could be responsible for miR-338-5p function.

Afterwards, we measured both mRNA expression and protein level of NFAT5 in transfected RAFLSs. Especially, NFAT5 was strongly inhibited by miR-338-5p mimic at both mRNA and protein level while miR-338-5p inhibitor promotes its expression dramatically $(P<0.05$, Fig. 4E and 4F). No significant change was found among blank control, negative control 1 and negative control 2groups. All the evidences supported our hypothesis that miR-338-5p functions on RAFLSs via downregulating NFAT5.

\section{MiR-338-5p promoted biological behaviors via downregulating NFAT5}

The low expression of NFAT5 mRNA in RA tissues and RAFLSs compared to relative healthy control was first confirmed using RT-qPCR $(P<0.05$, Fig. 5A and 5C). And Pearson correlation analysis indicated that NFAT5 mRNA expression in RA tissues was negatively correlated with miR-338-5p expression ( $r=-0.651, P<0.001$, Fig. 5B). Moreover, the protein level of NFAT5 also decreased in RA tissues and RAFLSs $(P<0.05$, Fig. 5D-5F). The aberrant expression of NFAT5 suggested a compact relationship among miR-338-5p, NFAT5 and RA. Hence, we divided RAFLSs into four groups: Blank control group (Blank) without any treatment, Negative control 1 group (NC) transfected with pcDNA3.1-NFAT5 cDNA, NFAT5

Fig. 5. Expression of NFAT5 in RA synovial tissues and RAFLSs. (A) NFAT5 mRNA expression was measured in 60 RA tissues and 30 healthy control tissues. (B) Linear analysis indicated that miR338-5p expression was negatively correlated with NFAT5 mRNA expression. Pearson

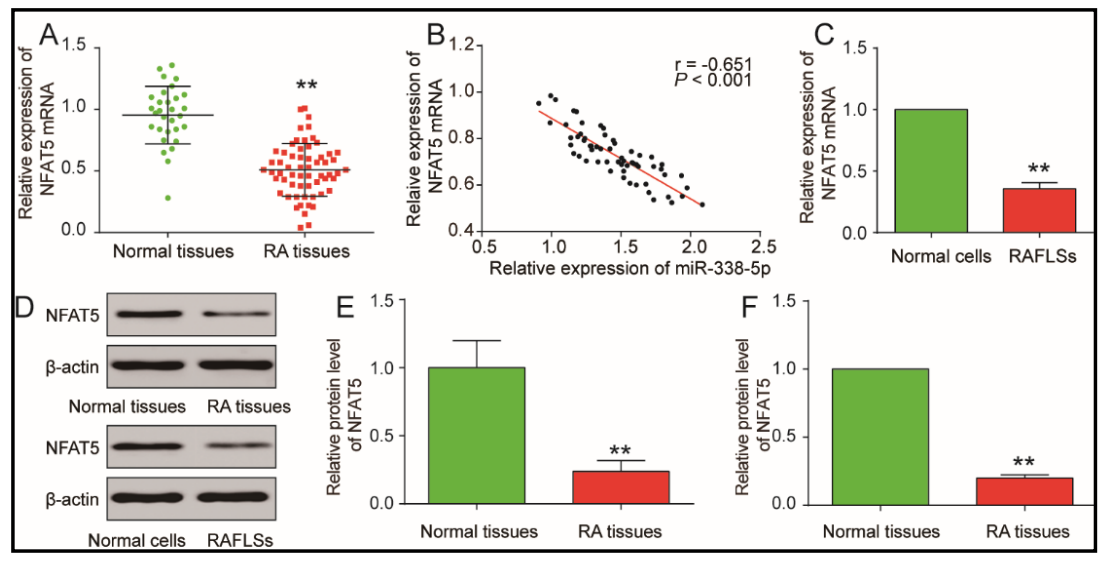
correlation analysis was used for data comparisons. (C) MRNA expression of NFAT5 in RAFLSs and healthy control cells were detected using RT-qPCR. (D-F) Protein level of NFAT5 in RA synovial tissues and RAFLSs was compared with the healthy control. Error bar indicates mean values \pm SD. ${ }^{* *} \mathrm{P}<0.01$. 


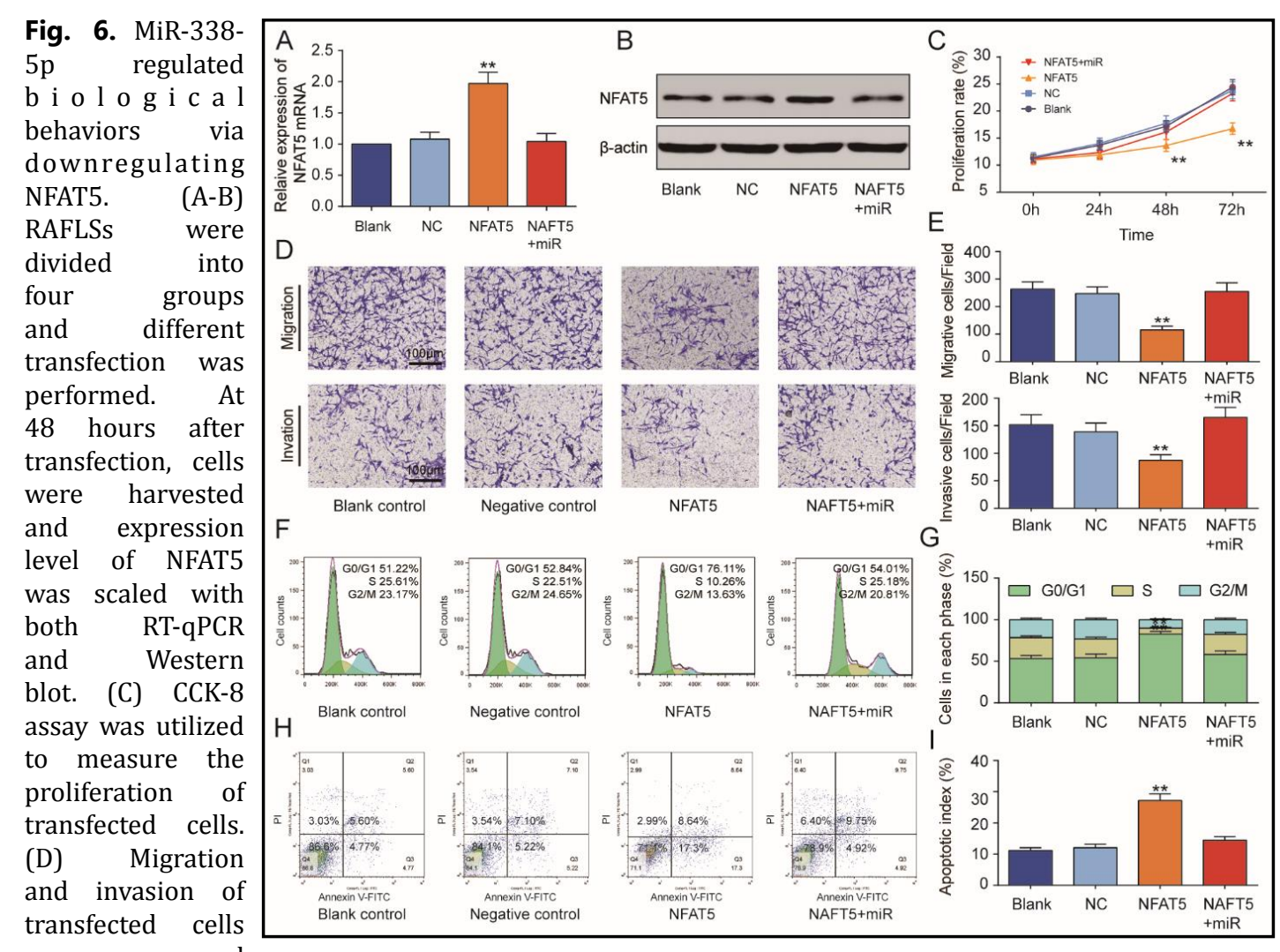

was measured

using Transwell assay. (E-F) Cell cycle and apoptosis rate was evaluated using flow cytometry. Error bar indicates mean values of triplicate experiments $\pm \mathrm{SD}$. ${ }^{* *} \mathrm{P}<0.01$.

group transfected with pcDNA3.1-NFAT5 cDNA and NFAT5 + miR group transfected with both pcDNA3.1-NFAT5 cDNA and miR-338-5p. RT-qPCR and western blot results confirmed the efficiency of transfection (Fig. 6A and 6B). NFAT5 expression in RAFLSs markedly increased in NFAT5 group compared to blank and NC group while co-transfection with miR$338-5 p$ reverse the amplification in NFAT5 + miR group $(P<0.05)$. Intriguingly, there is no significant difference among blank, NC and NFAT5 + miR group. Following assay including CCK-8, Transwell and Flow cytometry indicated that overexpression of NFAT5 impaired the cell viability, migratory and invasive abilities of RAFLSs compared to the control $(P<$ 0.05 , Fig. 6C-6E). These negative effects were attenuated by upregulation of miR-338-5p. Moreover, the G1/G0 arrest and enhancive cell apoptosis induced by exogenous NFAT5 could also be attenuated by co-transfection of miR-338-5p $(P<0.05$, Fig. 6F-6I). Taken together, sufficient evidences supported that NFAT5 could suppressed the proliferation, migration, invasion and blocked cellular progression from G2/M into G0/G1 of RAFLSs and miR-338-5p functions conversely on RAFLSs via downregulating RAFLSs.

\section{Discussion}

Various studies have identified the role of miRNAs in RA. For instance, Li Z's research suggested that miR-155 overexpressed in RAFLSs decreased MMP-3 levels and inhibited the proliferation and invasion of RAFLSs [31]. Ogando J et al. demonstrated that Notch signaling pathway is inhibited by the overexpression of miR-223 in RA patients [32]. With further efforts, Ogando and his collogues identified miR-223 as a negative regulator of AHR/ARNT pathway by targeting ARNT, which negatively modulates the downstream Notch effectors [32]. Yan C et al. identified mitomycin C induces apoptosis in rheumatoid arthritis fibroblast- 


\section{Cellular Physiology Cell Physiol Biochem 2018;49:899-910

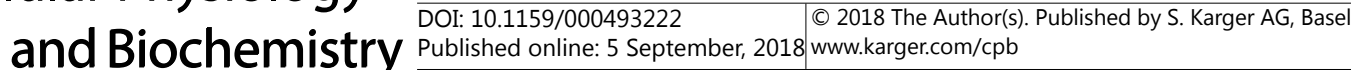 \\ Guo et al.: miR-338-5p Targets NFAT5 and Influences Raflss}

like synoviocytes [33]. In our study, the expression level of miR-338-5p in RA synovium was significantly higher than that in normal synovium. Our results suggested that the overexpression of miR-338-5p may promote the proliferation, migration and invasion of RAFLSs. The different expressions of miR-338-5p in rheumatoid arthritis could be due to the differentiated microenvironment of RA synovium.

The putative target of miR-338-5p, NFAT-5, also labeled as TonEBP or OREBP, has been reported to be involved in immune and inflammatory responses regulation as well as tumorigenesis [34]. The expression of NFAT-5 was repressed at both mRNA and protein levels in RA tissues and RAFLSs. Multifarious of priors have studied the influence of NFAT- 5 in different diseases. For example, Qin X et al. demonstrated that NFAT5 promotes apoptosis by regulating PARP-1, BAX/BCL2 while inhibits invasion through EMT-related protein claudin-1 and fibronectin [35]; Hao $S$ et al. indentified that NFAT5 is part of a protective mechanism that limits renal damage induced by IRI (ischemia-reperfusion injury) in experiment [36], which are similar to our findings. Our Luciferase assay confirmed that miR-338-5p directly targeted NFAT-5. Several reports conveyed the information that NFAT5 could help sustain intracellular homeostasis which is critical for natural cell proliferation [37,38]. Accumulating evidence suggests that NFAT-5 is attached to cytoskeleton and activate downstream effectors like Cox-2 to facilitate cancer cell metastasis [39]. In addition, NFAT signaling in the tumor microenvironment is likely to have a significant impact on chemokine signaling [40]. In this study, we investigated how NFAT- 5 was regulated by miR-338-5p and thereafter influenced RAFLSs in RA occurrence and development. These findings well supported our hypothesis that miR-338-5p may influence the proliferation, migration and the inflammation responses of RAFLSs by targeting NFAT5. We intended to focus on the interplay between NFAT- 5 and immune microenvironment and how their interaction regulates the RAFLS activities in the future studies. That would also contribute to understanding of how miR-338-5p influences the inflammatory responses in RA patients. Since sex and age of the RA patients may affect the development of RA, our experiment results have some limitations. Besides, the specific mechanism and functional analysis of miR-338 in cellular pathways as well as the influence of miR-338-5p on RA KEGG pathway still remains to be further studied. The expression of NFAT5 is related to changes in osmotic pressure, the expression of gene NFAT5 may not only connected with miR-338-5p, but with other genes or microenvironment, and needs to be further explored. Relative studies also shown NFAT5 knockdown inhibits hypertonicityinduced chondrogenic marker expression, including AGC1, SOX9, COL2 [41]. The concrete mechanism between NFAT5 and Rheumatoid arthritis may be NFAT5 influences the characteristic of RAFLSs by influencing the downstream gene.

\section{Conclusion}

Collectively, our experimental data demonstrated the significance of miR-338-5p activities in RAFLS and further elucidated the interaction between NFAT-5 and miR-338-5p. We illustrated that miR-338-5p could target NFAT5, and promote the RAFLSs proliferation and migration by for the first time. We thereafter identified NFAT5 as a critical player in RAFLS phenotypic modulation, and rendered a complete new perspective of the block of RAFLS migration in RA.

\section{Disclosure Statement}

The authors declare that they have no conflicts of interest concerning this article. 


\section{Cellular Physiology Cell Physiol Biochem 2018;49:899-910 \begin{tabular}{l|l|l} 
and Biochemistry Publisned onlIne: 5 september, 2018 & (c) 2018 The Author(s). Published by S. Karger AG, Basel \\
ww.karger.com/cpb
\end{tabular}}

Guo et al.: miR-338-5p Targets NFAT5 and Influences Raflss

\section{References}

1 Sharma AR, Sharma G, Lee SS, Chakraborty C: miRNA-Regulated Key Components of Cytokine Signaling Pathways and Inflammation in Rheumatoid Arthritis. Med Res Rev 2016;36:425-439.

-2 Niederer F, Trenkmann M, Ospelt C, Karouzakis E, Neidhart M, Stanczyk J, Kolling C, Gay RE, Detmar M, Gay S, Jungel A, Kyburz D: Down-regulation of microRNA-34a* in rheumatoid arthritis synovial fibroblasts promotes apoptosis resistance. Arthritis Rheum 2012;64:1771-1779.

-3 Duroux-Richard I, Pers YM, Fabre S, Ammari M, Baeten D, Cartron G, Touitou I, Jorgensen C, Apparailly F: Circulating miRNA-125b is a potential biomarker predicting response to rituximab in rheumatoid arthritis. Mediators Inflamm 2014;2014:342524.

-4 Trenkmann M, Brock M, Gay RE, Michel BA, Gay S, Huber LC: Tumor necrosis factor alpha-induced microRNA-18a activates rheumatoid arthritis synovial fibroblasts through a feedback loop in NF-kappaB signaling. Arthritis Rheum 2013;65:916-927.

5 Huber LC, Distler O, Tarner I, Gay RE, Gay S, Pap T: Synovial fibroblasts: key players in rheumatoid arthritis. Rheumatology (Oxford) 2006;45:669-675.

6 Schonfeld C, Pap T, Neumann E, Muller-Ladner U: [Fibroblasts as pathogenic cells in rheumatic inflammation]. Z Rheumatol 2015;74:33-38.

7 Noss EH, Brenner MB: The role and therapeutic implications of fibroblast-like synoviocytes in inflammation and cartilage erosion in rheumatoid arthritis. Immunol Rev 2008;223:252-270.

$>8$ Xu D, Yan S, Wang H, Gu B, Sun K, Yang X, Sun B, Wang X: IL-29 Enhances LPS/TLR4-Mediated Inflammation in Rheumatoid Arthritis. Cell Physiol Biochem 2015;37:27-34.

-9 Jiang S, Li Y, Lin T, Yuan L, Li Y, Wu S, Xia L, Shen H, Lu J: IL-35 Inhibits Angiogenesis through VEGF/Ang2/ Tie2 Pathway in Rheumatoid Arthritis. Cell Physiol Biochem 2016;40:1105-1116.

10 Nakasa T, Miyaki S, Okubo A, Hashimoto M, Nishida K, Ochi M, Asahara H: Expression of microRNA-146 in rheumatoid arthritis synovial tissue. Arthritis Rheum 2008;58:1284-1292.

-11 Nakamachi Y, Kawano S, Takenokuchi M, Nishimura K, Sakai Y, Chin T, Saura R, Kurosaka M, Kumagai S: MicroRNA-124a is a key regulator of proliferation and monocyte chemoattractant protein 1 secretion in fibroblast-like synoviocytes from patients with rheumatoid arthritis. Arthritis Rheum 2009;60:1294-1304.

12 Kloosterman WP, Plasterk RH: The diverse functions of microRNAs in animal development and disease. Dev Cell 2006;11:441-450.

13 Stanczyk J, Ospelt C, Karouzakis E, Filer A, Raza K, Kolling C, Gay R, Buckley CD, Tak PP, Gay S, Kyburz D: Altered expression of microRNA-203 in rheumatoid arthritis synovial fibroblasts and its role in fibroblast activation. Arthritis Rheum 2011;63:373-381.

14 Ceribelli A, Nahid MA, Satoh M, Chan EK: MicroRNAs in rheumatoid arthritis. FEBS Lett 2011;585:36673674.

15 Ceribelli A, Yao B, Dominguez-Gutierrez PR, Nahid MA, Satoh M, Chan EK: MicroRNAs in systemic rheumatic diseases. Arthritis Res Ther 2011;13:229.

-16 Mookherjee N, El-Gabalawy HS: High degree of correlation between whole blood and PBMC expression levels of miR-155 and miR-146a in healthy controls and rheumatoid arthritis patients. J Immunol Methods 2013;400-401:106-110.

17 Filkova M, Jungel A, Gay RE, Gay S: MicroRNAs in rheumatoid arthritis: potential role in diagnosis and therapy. BioDrugs 2012;26:131-141.

-18 Kos A, Olde Loohuis NF, Wieczorek ML, Glennon JC, Martens GJ, Kolk SM, Aschrafi A: A potential regulatory role for intronic microRNA-338-3p for its host gene encoding apoptosis-associated tyrosine kinase. PLoS One 2012; 7:e31022.

19 Yong FL, Law CW, Wang CW: Potentiality of a triple microRNA classifier: miR-193a-3p, miR-23a and miR338-5p for early detection of colorectal cancer. BMC Cancer 2013;13:280.

20 Takei Y, Ohnishi N, Kisaka M, Mihara K: Determination of abnormally expressed microRNAs in bone marrow smears from patients with follicular lymphomas. Springerplus 2014;3:288.

21 Chang H, Kim N, Park JH, Nam RH, Choi YJ, Lee HS, Yoon H, Shin CM, Park YS, Kim JM, Lee DH: Different microRNA expression levels in gastric cancer depending on Helicobacter pylori infection. Gut Liver 2015;9:188-196.

-22 Baumgart BR, Gray KL, Woicke J, Bunch RT, Sanderson TP, Van Vleet TR: MicroRNA as biomarkers of mitochondrial toxicity. Toxicol Appl Pharmacol 2016;312:26-33. 


\section{Cellular Physiology Cell Physiol Biochem 2018;49:899-910 and Biochemistry DOI: 10.1159/000493222 1 (c) 2018 The Author(s). Published by S. Karger AG, Basel

23 Klareskog L, Padyukov L, Ronnelid J, Alfredsson L: Genes, environment and immunity in the development of rheumatoid arthritis. Curr Opin Immunol 2006;18:650-655.

-24 Klareskog L, Ronnelid J, Lundberg K, Padyukov L, Alfredsson L: Immunity to citrullinated proteins in rheumatoid arthritis. Annu Rev Immunol 2008;26:651-675.

25 Ma X, He X, Dong P, Xue D, Song D, Xu H, Zhang X: [miR-338-5p modulates B cell biological functions by targeting NF-kappaB1]. Xi Bao Yu Fen Zi Mian Yi Xue Za Zhi 2016;32:1475-1480.

-26 Kim NH, Choi S, Han EJ, Hong BK, Choi SY, Kwon HM, Hwang SY, Cho CS, Kim WU: The xanthine oxidaseNFAT5 pathway regulates macrophage activation and TLR-induced inflammatory arthritis. Eur J Immunol 2014;44:2721-2736.

-27 Masuda K, Masuda R, Neidhart M, Simmen BR, Michel BA, Muller-Ladner U, Gay RE, Gay S: Molecular profile of synovial fibroblasts in rheumatoid arthritis depends on the stage of proliferation. Arthritis Res 2002;4:R8.

28 Tardif G, Pelletier JP, Fahmi H, Hum D, Zhang Y, Kapoor M, Martel-Pelletier J: NFAT3 and TGF-beta/SMAD3 regulate the expression of miR-140 in osteoarthritis. Arthritis Res Ther 2013;15:R197.

29 Jauliac S, Lopez-Rodriguez C, Shaw LM, Brown LF, Rao A, Toker A: The role of NFAT transcription factors in integrin-mediated carcinoma invasion. Nat Cell Biol 2002;4:540-544.

30 Yoon HJ, You S, Yoo SA, Kim NH, Kwon HM, Yoon CH, Cho CS, Hwang D, Kim WU: NF-AT5 is a critical regulator of inflammatory arthritis. Arthritis Rheum 2011;63:1843-1852.

-31 Long L, Yu P, Liu Y, Wang S, Li R, Shi J, Zhang X, Li Y, Sun X, Zhou B, Cui L, Li Z: Upregulated microRNA-155 expression in peripheral blood mononuclear cells and fibroblast-like synoviocytes in rheumatoid arthritis. Clin Dev Immunol 2013;2013:296139.

-32 Ogando J, Tardaguila M, Diaz-Alderete A, Usategui A, Miranda-Ramos V, Martinez-Herrera DJ, de la Fuente L, Garcia-Leon MJ, Moreno MC, Escudero S, Canete JD, Toribio ML, Cases I, Pascual-Montano A, Pablos JL, Manes S: Notch-regulated miR-223 targets the aryl hydrocarbon receptor pathway and increases cytokine production in macrophages from rheumatoid arthritis patients. Sci Rep 2016;6:20223.

-33 Yan C, Kong D, Ge D, Zhang Y, Zhang X, Su C, Cao X: Mitomycin C induces apoptosis in rheumatoid arthritis fibroblast-like synoviocytes via a mitochondrial-mediated pathway. Cell Physiol Biochem 2015;35:11251136.

34 Mancini M, Toker A: NFAT proteins: emerging roles in cancer progression. Nat Rev Cancer 2009;9:810-820.

35 Qin X, Wang Y, Li J, Xiao Y, Liu Z: NFAT5 inhibits invasion and promotes apoptosis in hepatocellular carcinoma associated with osmolality. Neoplasma 2017;64:502-510.

-36 Hao S, Bellner L, Zhao H, Ratliff BB, Darzynkiewicz Z, Vio CP, Ferreri NR: NFAT5 is protective against ischemic acute kidney injury. Hypertension 2014;63:e46-52.

-37 Yoeli-Lerner M, Chin YR, Hansen CK, Toker A: Akt/protein kinase b and glycogen synthase kinase-3beta signaling pathway regulates cell migration through the NFAT1 transcription factor. Mol Cancer Res 2009;7:425-432.

-38 Arroyo JA, Garcia-Jones P, Graham A, Teng CC, Battaglia FC, Galan HL: Placental TonEBP/NFAT5 osmolyte regulation in an ovine model of intrauterine growth restriction. Biol Reprod 2012;86:94.

-39 Siamakpour-Reihani S, Caster J, Bandhu Nepal D, Courtwright A, Hilliard E, Usary J, Ketelsen D, Darr D, Shen XJ, Patterson C, Klauber-Demore N: The role of calcineurin/NFAT in SFRP2 induced angiogenesis--a rationale for breast cancer treatment with the calcineurin inhibitor tacrolimus. PLoS One 2011;6:e20412.

40 Medyouf H, Alcalde H, Berthier C, Guillemin MC, dos Santos NR, Janin A, Decaudin D, de The H, Ghysdael J: Targeting calcineurin activation as a therapeutic strategy for T-cell acute lymphoblastic leukemia. Nat Med 2007;13:736-741.

41 van der Windt AE, Haak E, Das RH, Kops N, Welting TJ, Caron MM, van Til NP, Verhaar JA, Weinans H, Jahr $\mathrm{H}$ : Physiological tonicity improves human chondrogenic marker expression through nuclear factor of activated T-cells 5 in vitro. Arthritis Res Ther 2010;12:R100. 